\title{
A MULTI-PARADIGM, WHOLE SYSTEM VIEW OF HEALTH AND SOCIAL CARE FOR AGE-RELATED MACULAR DEGENERATION
}

\author{
Joe Viana \\ Stuart Rossiter \\ Andrew A. Channon \\ Sally C. Brailsford \\ Andrew Lotery \\ University of Southampton \\ University Road \\ Southampton SO17 1BJ, UNITED KINGDOM
}

\begin{abstract}
This paper presents a hybrid simulation model for the management of an eye condition called age-related macular degeneration, which particularly affects the elderly. The model represents not only the detailed clinical progression of disease in an individual, but also the organization of the hospital clinic in which patients with this condition are treated and the wider environment in which these patients live (and their social care needs, if any, are met). The model permits a 'whole system' societal view which captures the interactions between the health and social care systems.
\end{abstract}

\section{INTRODUCTION}

This paper models the treatment and progression of age-related macular degeneration (AMD) - a common form of visual impairment in the elderly - in the UK county of Hampshire, working with the Southampton General Hospital Eye Unit. Although at its core a classic OR problem in improving the efficiency of the clinic, the model attempts a whole system view which incorporates the relationships between health and social care for sufferers, and related cross-service planning and budgeting issues for the stakeholders. This suggests the need for a hybrid model using discrete-event simulation (DES), agent-based modeling $(\mathrm{ABM})$ and system dynamics (SD), chosen so as to use the 'best tools for the job' rather than rigidly sticking to one paradigm. Both this methodological fusion and the whole system view are overarching themes of the Care Life Cycle (CLC) project which the work is a part of (Care Life Cycle 2012); these themes are echoed in the other CLC-related papers at this conference (Brailsford et al. 2012; Noble et al. 2012).

\subsection{Clinical \& Social Context}

Visual impairment is a common form of disability amongst the elderly in the UK, with estimates of the numbers over 65 suffering from some form of impairment ranging from $1.6 \mathrm{~m}$ to $2.2 \mathrm{~m}$ (Charles 2007). The most common cause of visual impairment in this age group is AMD, a progressive degenerative disease that causes the loss of central vision (The Eye Diseases Prevalence Research Group 2004). Due to the increasing older adult population over the age of 65 in the UK it is predicted that the numbers with AMD will increase, from just over 600,000 in 2010 to over 750,000 in 2020 (Minassian, Reidy et al. 2011). The estimated cost of this disability, in detection, treatment and the provision of care is $£ 1.6$ billion per year (Minassian and Reidy 2009). 
There are many forms of AMD, although they are broadly grouped as being either 'wet' and 'dry', with 'wet' AMD consisting of between $65-70 \%$ of the total disability burden (Minassian et al. 2011). The 'dry' form of AMD is irreversible and is characterized by a slow transition to impairment, while the 'wet' form results in more rapid visual deterioration. However it can usually be treated using injections of ranibizumab which slows the impairment or even causes vision to improve (Lotery 2008). These injections need to be given at regular monthly intervals in order to show benefit to the patient. This is a new treatment and many hospital eye units do not have the organizational structure nor the capacity to manage the regular need for injections or consulting. Appointment booking systems are not efficient at managing this regular need for care.

The implications of visual impairment in general, and AMD in particular, are wide-ranging. Those with AMD are seen to be more likely to be depressed (Brody et al. 2001), have a lower quality of life (Slakter and Stur 2005; Hassell et al. 2006) and less functional independence (Williams et al. 1998). It is estimated that mild AMD causes a similar reduction in the quality of life as moderate cardiac angina (Brown et al. 2005). The reduced vision interferes with the ability to self-care (Stevenson, Hart et al. 2004), leading to increasing reliance on both formal (i.e., provided through an established channel) and informal (i.e., given by family or friends) social care. Without this care the individual may not be able to function on their own and require a higher level of health care for non-visual related health issues.

The Eye Unit at Southampton General Hospital is the sole treatment center for AMD in the UK county of Hampshire. It is currently providing injections for those with wet AMD but it is not felt that this is being conducted in the most efficient manner. Furthermore there is concern about the ability of the Unit to cope with the growing ageing population, which could potentially lead to a number of patients failing to receive the care that they require, increasing the social care burden.

This paper focuses on the development and treatment of 'wet' AMD in the area served by the Southampton Eye Unit. Sufferers of the condition have related care needs, often complicated by other conditions due to the age-related nature of AMD. These needs may or may not be met through receiving social care, either formally (provided by the state or privately) or informally (provided by family and friends). Formal social care is determined after an assessment of need while informal care depends on the network of friends and family surrounding the individual. The social care obtained also links with the ease of travelling to Southampton General Hospital for treatment - those with little support will find this more difficult and may miss appointments leading to further deterioration of vision, enhancing the need for social care. The Eye Unit needs to be organized to allow treatment of the maximum numbers of patients without reducing the patient experience.

The study has been carried out in collaboration with the Southampton Eye Unit in order to explore alternative configurations of the clinic in the safety of a computer environment, including potential options such as mobile eye unit which perform time-consuming eye photography to alleviate pressure on shared facilities at the clinic. Through exploring different potential configurations of care given at the Eye Unit, changes in the efficiency of the Unit can be estimated as well as the corresponding improvements in health and reductions in the need for social care. Ultimately, the work also aims to highlight how expenditure in one area (health or social care) may cause non-obvious cost implications in the other (e.g., improved social care transport services saving considerable amounts in the clinic due to less missed appointments, and with knock-on savings in social care due to less avoidable AMD sight loss).

\section{METHODOLOGY}

Figure 1 illustrates the conceptual architecture of the model, which is not yet fully realized in the current implementation (section 3) but guides the work. DES and SD components sit inside an overarching agentbased model. Individuals (agents) in the population may develop AMD, alongside other conditions, and have varying levels of care need. Some may provide informal care to others. Embedded SD models in each individual model progressive sight loss from AMD and other conditions. Individuals with eye conditions (not just AMD) will interact with the healthcare system via the ophthalmology department and, potentially, mobile units. The clinic is modeled as a DES with explicit appointment scheduling processes. 
Doctors interact with this clinic, and may respond to scheduling pressures (e.g., by adjusting the slots in their timetables for different conditions).

Interactions occur in a spatial environment, which can be used to model aspects such as ability to travel to the clinic, the location of those providing care to AMD sufferers (which influences their ability to provide care), and the positioning of mobile eye units.

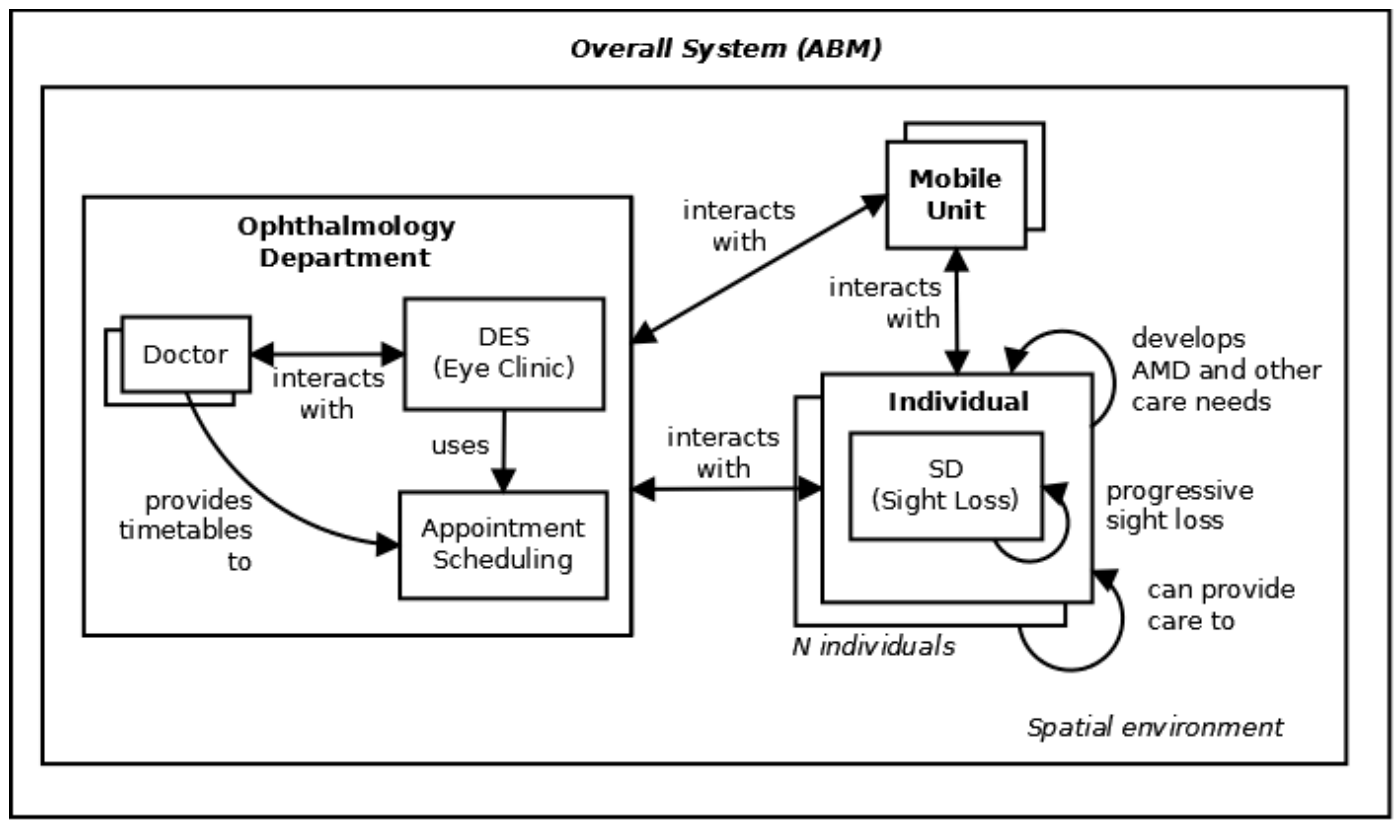

Figure 1: The overall conceptual architecture for the AMD model.

The hybridization in the model here can be seen as an elaborate combination of "processenvironment" type combinations (Chahal and Eldabi 2008), if their focus on DES and SD is relaxed to include ABM. Sub-models sit inside other models, with the inner model representing a process which interacts with the wider system environment of the outer model. A similar approach was used in the two illustrative examples of Brailsford et al. (2010). In Swinerd and McNaught's terminology for ABM and SD hybrids (2012), the model uses flavors of their "integrated" hybrid design alternative (cf. "interfaced" or "sequential"), with a similar need to relax the specific focus.

he modeling here is primarily motivated by using the best combination of tools, rather than any claim of novel hybridization. By 'best', we mean using the modeling paradigm which most cleanly implements the conceptual design we had in mind for each sub-system. This is discussed per sub-system in section 3. By combining the different methodologies which are best suited for their particular tasks, the stakeholders have gained greater buy-in and understanding, where the stakeholders include both the problem owners (health care and social care professionals) and those members of the project team who are unfamiliar with the techniques. In technical terms, the modeling framework used (AnyLogic) natively provides constructs with which to link the paradigms; some simple additional 'plumbing' code was still needed to allow agents to pass through the DES wrapped in a DES entity object.

\subsection{Stakeholder Interaction}

The model was developed with input from staff at the Eye Unit, but not in a fully stakeholder-driven process. The DES models the clinic at a fine-grained time resolution, but the long-term effects of treatments on sight loss are also of interest, particularly with feedback effects due to changes in social care and its influence on missed injections. These long timescales mean that assumptions have to be made about what 
is subject to change and what can be regarded as static. The emphasis therefore has to be on presenting challenging 'what-if' scenarios, and not 'best guess' projections or predictions.

\subsection{Scenarios}

There are a wide range of potential 'what-if' scenarios that could be explored within this conceptual framework. In general, the clinic DES model (section 3.2) and sight loss SD models ( section 3.3) can be used to explore health care scenarios, and the agent-based elements (section 3.4) to explore social care scenarios. The social care consequences can be evaluated in terms of health care scenarios, and vice versa. Examples of potential scenarios which could be evaluated are shown in Table 1.

Table 1: Potential scenarios for evaluation.

\begin{tabular}{|l|l|}
\hline Health Care & Social Care \\
\hline $\begin{array}{l}\text { Ophthalmology department efficiency } \\
\text { Patient pathways, opening times, ap- } \\
\text { pointment times, availability of resources } \\
\text { both staff and medical facilities for ex- } \\
\text { ample. }\end{array}$ & $\begin{array}{l}\text { Social care need } \\
\text { Changing development rates for varying } \\
\text { levels of care need in relation to age, } \\
\text { and the specific impact of AMD on the- } \\
\text { se rates. }\end{array}$ \\
\hline $\begin{array}{l}\text { Sight loss and AMD Stages } \\
\begin{array}{l}\text { Differing models of sight deterioration } \\
\text { (both natural and AMD), and the effects } \\
\text { of differing treatments (including indi- } \\
\text { vidual heterogeneity in response). }\end{array}\end{array}$ & $\begin{array}{l}\text { Social care provision } \\
\text { Changes in the patterns of care provided } \\
\text { to those in need (both level and type). }\end{array}$ \\
\hline
\end{tabular}

For the current paper the aim is to demonstrate the interconnectedness between the health and social care systems, highlighting how changes in one sphere can result in changes to the other. Four different scenarios are therefore investigated, representing: the current situation, changes to the healthcare supply, changes to the social care supply, and a combination of the two. These will be fully explained in section 4.2.

\section{DETAILS OF THE MODEL}

The current model represents a stepping-stone towards the full aspirations highlighted in section 2 . Most areas have been parameterized with accurate data from various sources, the main ones being: the English Longitudinal Study of Ageing (ELSA 2012), the Age-Related Eye Disease Study Group (AgeRelated Eye Disease Study Research 2000; Age-Related Eye Disease Study Research 2005), the Royal National Institute of Blind People (Access Economics PTY Limited 2009), and information directly obtained from the Eye Unit regarding operation and performance. Fuller details can be found at http://www.soton.ac.uk/clc/publications/supplementary, together with the parameters used from these data sources in different parts of the model.

The model is implemented in AnyLogic (www.xjtek.com/AnyLogic), a commercial package that supports the integration of DES, SD and agent-based models, with features for each comparable to paradigm-specific products.

\subsection{Overall Structure}

Individuals develop AMD, and will then interact with the DES that represents the eye clinic via appointments (see Figure 2). 
Viana, Rossiter, Channon, Brailsford, and Lotery

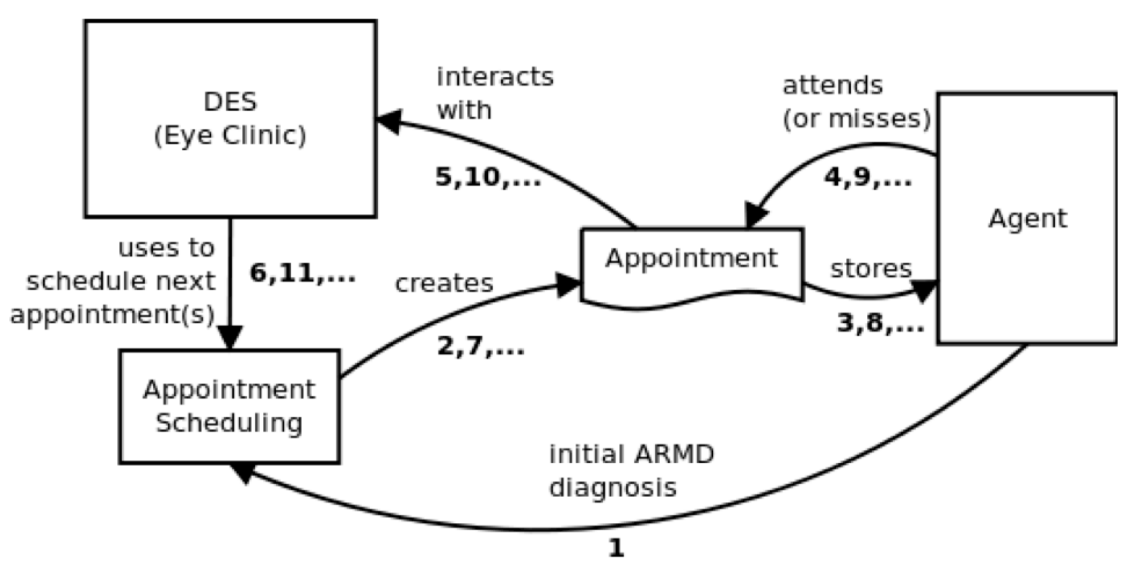

Figure 2: The main per-individual dynamics of interaction with the eye clinic. Numbers indicate the sequence of operations.

On an initial AMD diagnosis, the individual (agent) is scheduled for an initial appointment and stores this information, effectively setting up an event for the appointment time. The patient then attends the appointment, at which point it interacts with the eye clinic DES. After the appointment is finished the agent leaves the DES model and the next appointment, which may be either a follow-up or an injection appointment, is scheduled This scheduling currently uses some simplifying assumptions about the number and duration of available slots, but still captures the essence of the real-life mechanism. This dynamic cycle of appointments continues until other events, such as death, intervene. The treatment that the individual may or may not receive impacts the progression of his/her sight loss in the agent-embedded SD models.

Individuals can miss appointments for a variety of reasons (such as transport difficulties, illness or simply through forgetfulness) and can fail to complete appointments (generally due to excessive queues in the clinic, or due to insufficiently flexible transport arrangements). In both cases, an injection is missed which will cause avoidable sight deterioration. These are the main mechanisms by which social care issues can influence the clinic's operation. Missed appointments are modeled as probabilities based on the individual's care need and the type of care received (where need and provision levels map to multipliers applied to a basic fixed probability of missing the appointment). Incomplete appointments are modeled as probabilities based on time spent queuing. These parameters are currently based on expert opinion, but there is potential to use empirical data to improve them.

\subsection{Eye Clinic DES Model}

The choice of DES to model the Eye Unit is natural to simulate the detailed processes and layout of the system. Clearly this is unique to the context, but in overall structure and concept it is a standard DES outpatient clinic model in a long tradition of such queuing network type models. Jun et al. (1999) present a survey of 117 such clinic models, although obviously not all for eye conditions, and this is a classical application area for DES in health.

DES has also been applied, unusually, for modeling the clinical progression of eye disease by Davies et al. (2000; 2002), in order to evaluate the effectiveness of different screening policies for diabetic retinopathy. The approach most typically used to model clinical progression in economic evaluations of interventions is either Markov modeling or decision tree analysis (Cooper, Brailsford and Davies 2007). In the specific case of AMD, there are a small number of published models which use models to study the cost-effectiveness or cost-utility of different drugs, for example Fletcher et al. (2008), but none that use DES.

Staff from the clinic have been involved in the conceptualization of the model, helping to define the patient pathways and physical layout of the clinic. AnyLogic allows the entities to be modeled physically 
moving through the clinic space as they go through the steps of the pathway. The results shown here use modeler estimates for the parameters. The clinic is hoping to make internal tracking data available soon, which can be used to derive more empirically-driven values. A patient questionnaire will also be incorporated to aid the parameterization of the patients' behavior outside the clinic, such as their mode of transport to the clinic, who accompanied them to the clinic and care relationships.

Full details of the DES model structure and data are available on our project Web site at http://www.soton.ac.uk/clc/publications/supplementary.

\subsection{Sight Loss and AMD Progression (State-Transition and Embedded SD Models)}

The process of sight loss, both natural and AMD-related, lends itself to a rate-based mathematical representation which SD models are ideal for. (In our case, 'natural' could conceptually include other nonAMD eye conditions.) Sight level is modeled very simply as a stock which is decreased via natural and AMD loss rates. The progression of AMD itself is modeled by a simple set of stochastic stage transitions (using AnyLogic statecharts), where the stages cause changes in the AMD-related sight-loss rate. AMD does not affect each eye identically. As a result, sight loss progresses differently in each eye and hence each agent actually has a pair of SD models (one for each eye), each parameterized separately. Figure 3 shows a schematic of the SD model used for the left eye; the model for the right eye is the same. Obtaining an injection will slow the AMD-related degeneration of sight (Lotery 2008).

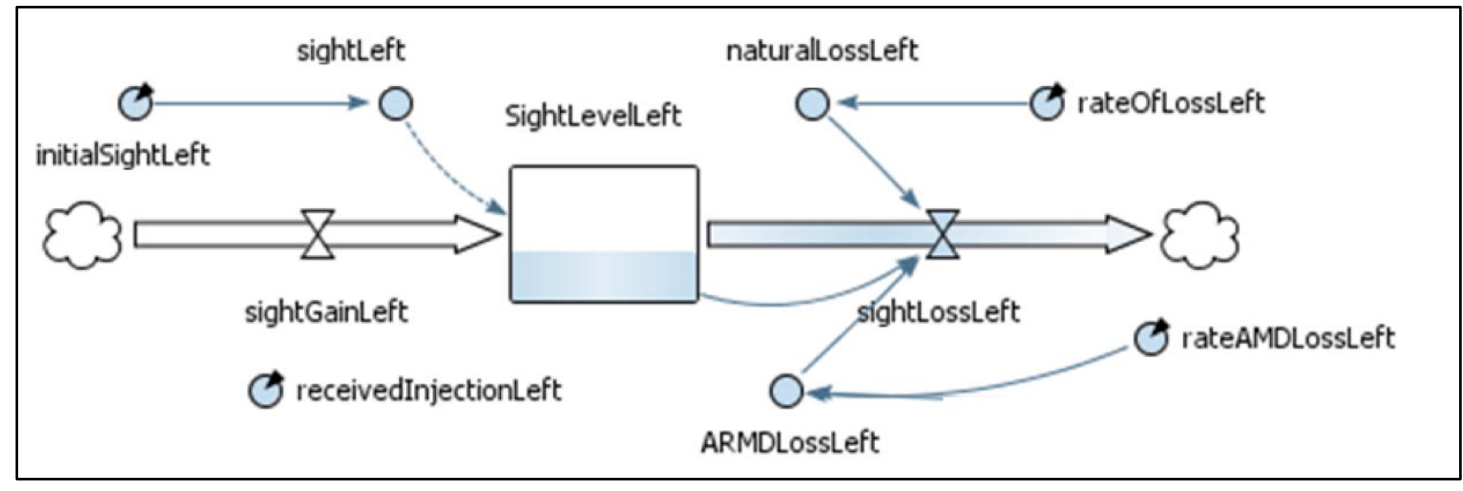

Figure 3: Schematic of the SD model for an eye.

There is individual heterogeneity, both for each eye and between individuals, including in their reaction to injections and the 'virulence' of their AMD. Future work will align this sub-model more explicitly with specific clinical details of sight loss and AMD progression (e.g., Klein et al. 2002; Owen et al. 2012).

\subsection{Individual Care, Mortality and AMD Development}

Conceptually (as described in Figure 1), the model has a full population of individuals who will develop AMD and form care relationships. Implementing this in an empirically accurate way via explicit, endogenous agent behavior is a major challenge, and is an area of research in its own right. (It is typically done via regression-model-driven microsimulation, but see Noble et al. (2012) for other approaches being considered on the CLC project.)

To simplify matters, the current model abstracts away this detail. Only AMD-suffering individuals are explicitly included in the population, and are created synthetically based on current numbers of new patients, with incidence data used to set initial attributes such as age and gender. (These therefore represent patients already referred.) Dynamically changing care needs are modeled using statecharts, with the type of care provided stochastically derived from care need level. Transitions are based on cross-tabulated population-level statistics. An individual's AMD progression is linked to care need by having sight-level 
categories imposing a minimum level of care need (with some stochastic variation). Mortality is based on population-level age-dependent life tables, with per-year checks, and is a significant factor since at least $30 \%$ of AMD diagnoses are for $90+$ year-olds.

The accuracy of these parameters is currently limited by the fact that population-level data should ideally be adjusted so that it reflects distributions for AMD-sufferers in particular. This is still work in progress.

\subsection{Current Output Measures}

The current main output measures available from the model are as below:

\begin{tabular}{|l|l|}
\hline Medical (DES) & Health and Social (ABM, SD) \\
\hline Length of stay in the clinic. & AMD-suffering population size and mortality. \\
\hline $\begin{array}{l}\text { Numbers of missed injections (due to missed ap- } \\
\text { pointments or leaving appointments early). }\end{array}$ & AMD stage distribution in the population. \\
\hline $\begin{array}{l}\text { Numbers of patients treated and quality criteria for } \\
\text { timeliness of appointments. }\end{array}$ & $\begin{array}{l}\text { Care provision types distribution in the popula- } \\
\text { tion. }\end{array}$ \\
\hline $\begin{array}{l}\text { Utilization of Ophthalmology resources (rooms, } \\
\text { staff, waiting areas, etc.). }\end{array}$ & Sight level distribution in the population. \\
\hline & Care need level distribution in the population. \\
\hline
\end{tabular}

\section{EXPERIMENTATION}

As discussed in section 2.2, four 'what-if' scenarios were run in the current model to explore the interconnectedness of the health and social care systems. For the purposes of this paper, the number of missed injections was chosen as the main output measure for discussion (see section 4.3), since this is a rough proxy both for the efficiency of the healthcare system and the effectiveness of care provision in the community.

This exploration was originally intended to include extensive analysis of and the sensitivity of several outcome measures to key parameters, as well as using an initial and ongoing AMD-suffering population that matched the clinic's current levels: around 1600 'AMD eyes', which thus maps to 800-1600 individuals and, at least at some stages, 20 new patients a week. Run lengths of 30 years were planned so that the longer-term dynamics of sight loss and ageing could be explored. Unfortunately, even with considerable performance tuning, models with only 50 initial individuals and 2 new patients a week (on average) would take over 28 hours to run 15 simulated years on a relatively high specification system (AMD Phenom II at $3.1 \mathrm{GHz}$ ).

The current results are therefore scaled back to representative runs for 1 simulated year for each scenario, with 50 initial individuals and 2 new patients a week. The original analysis can hopefully be performed in future, via further performance tuning and/or increased computing resources and timescales.

\subsection{Scenarios Tested}

These are:

1. Base - The Eye Unit appointment system and organization remains as it currently is for the entire simulation duration. Data for the other health and social aspects of the model remain constant, drawn from the literature, data analysis of surveys, expert opinion and educated guesses. This is the scenario against which all others will be compared. 
2. Healthcare - In this scenario an additional Optical Coherence Tomography (OCT) machine is installed in the Eye Unit. After initial analysis and discussions with clinical members of the Unit, it was observed that this is the key area where current bottlenecks are observed in the system.

3. Social care - As not all individuals who need social care currently receive it, this scenario simulates some nominal care provision stimulus. Individuals have a $50 \%$ chance of receiving one 'level' better care than they would have previously, where the care provision types None, Informal, Formal and Both are viewed as an increasing sequence of levels (and with increasingly less negative effects on the likelihood of missing clinic appointments).

4. Healthcare and Social Care - this scenario combines scenarios 2 and 3 above.

\subsection{Results}

The results from single representative runs are shown in Figure 1. They suggest that improving the Eye Unit's capability by increasing the number of OCT machines results in more patients successfully completing their appointments, as fewer patients leave the department who have waited an excessive amount of time. Increasing the amount of social care also has a positive effect when compared with the base, with more patients being able to attend their appointments. What is somewhat counterintuitive at this stage is that the combined scenario leads to less reduction in numbers of missed appointments over time than the individual scenarios on their own. A possible explanation for this is that the increased appointment attendance from the social care scenario places more pressure on the clinic than the extra OCT (healthcare scenario) can alleviate. Thus, reduced missed appointments are outweighed by increased incomplete appointments.

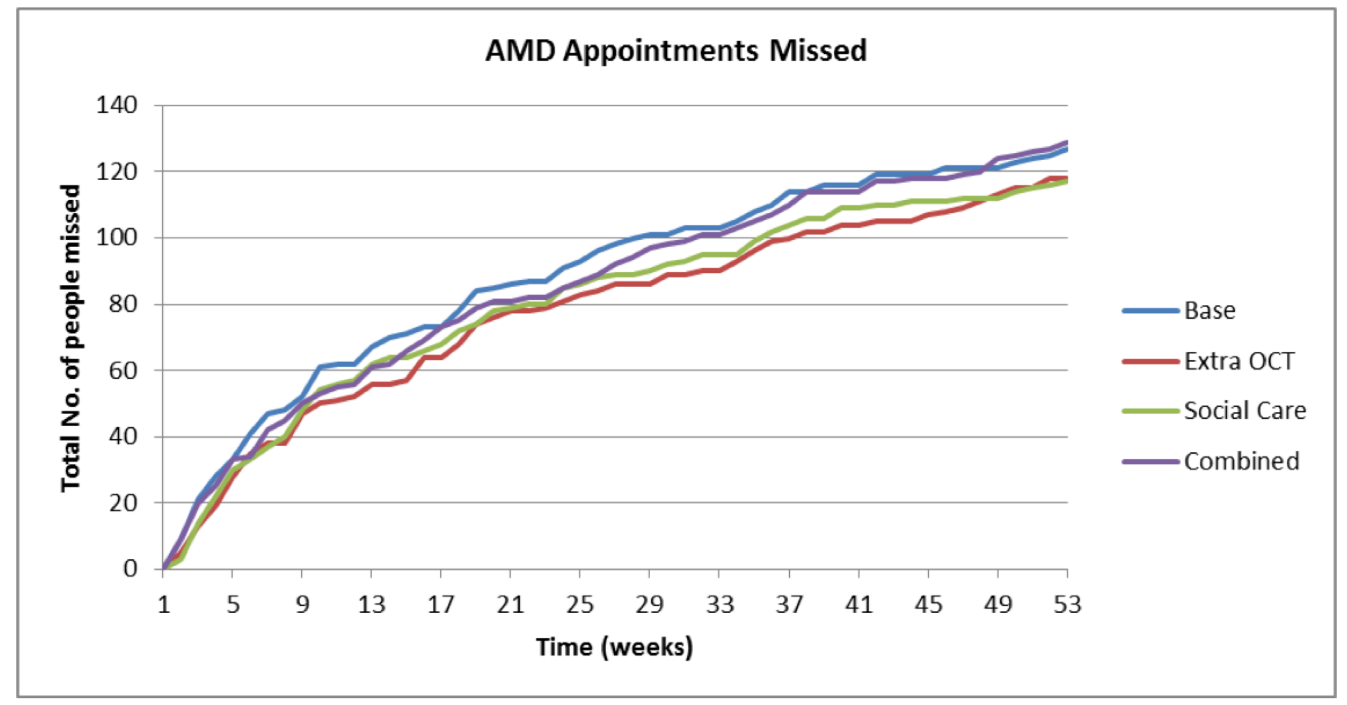

Figure 4: Results from initial experiments for the four scenarios.

\section{FUTURE WORK}

Future areas of work have been hinted at in the conceptual design (figure 1) and in the detail of the model and current results. These fall into the following broad categories:

- performance analysis of the model and the underlying AnyLogic platform;

- better quality data, reflecting the distributional specifics of the AMD-suffering population; 
- more accurate demographic processes (e.g., factoring in demographic change to the number of new AMD cases);

- a more empirically-validated sight loss sub-model, with related quality-adjusted life year (QALY) style metrics for avoidable sight-years lost due to system inefficiencies;

- more accurate reflection of the appointment scheduling process used;

- adding more nuanced detail of links between health and social care (e.g., inflexible hospitalprovided transport causing patients to have to leave appointments early, and better alignment of AMD progression with care need);

- increased spatiality, especially as regards scenarios for mobile eye-photography units;

- more 'ABM-like' explicit agent behavior, both for individuals in the population and doctors at the clinic, which can endogenously generate patterns of care provision and the like;

- estimates of the resultant impacts on costs in both the health and social care sectors (which will involve additional stakeholders).

\section{CONCLUSIONS}

This work has created a useful whole system modeling framework for health and social care. The aim of the CLC project is to take this whole system view, looking at each of the quadrants in Figure 5. There are many links between each of these areas, and this model has highlighted some of these for one disease (AMD). Focusing on a single disease has helped reduce the inevitable complexity, but the generality of some of the concepts and design suggests extensions beyond AMD. The project aims to use the right tools for the job (particularly ideas from complexity science) to demonstrate and understand the interactions and feedback between health and social care. The model here uses different components, constructed for specific purposes, which are then combined into a complete model. As a result it is easier to discuss it with stakeholders as they can focus on the area that is of most interest to them.

\begin{tabular}{l|c|c} 
& Demand & Supply \\
\hline Health Care & A & B \\
\hline Social Care & C & D
\end{tabular}

Figure 5: The quadrants of interest to the CLC project, reflecting the demand and supply of health and social care.

More generally, stakeholders can get 'traditional' OR advice on operational improvements whilst emphasizing the broader societal context, especially the sometimes-subtle effects of healthcare decisions on social care (and vice versa). This will be particularly useful going forwards, since the longer-term aim is to involve budget-holding stakeholders from health and social care, allowing them to see the broader implications and mutuality of their decisions. In England, the health and social care budgets are currently totally separate. Healthcare is funded by the taxpayer: it is provided by the National Health Service (NHS) and is free to the patient at the point of delivery. On the other hand, social care is provided by several different sources: informal care provided unpaid by family members or friends, and formal care provided either by the local county authority where the person lives, the private sector, or the voluntary sector. This model demonstrates the politics involved in a situation where relatively short-term expenditure by the NHS (e.g., providing an extra OCT machine in the clinic) can lead to longer-term cost savings for the local authority, since fewer people will go blind and require expensive residential care. Preventing avoidable sight loss in the elderly not only makes sense from a humanitarian perspective, it also makes sense from an economic perspective. However, without a whole system model which takes a societal perspec- 
tive, it is difficult to see why the NHS should bear the entire cost. The model provides a mechanism to explore such trade-offs.

The integration of DES, ABM and SD in a health care context is rare. This study adds to the knowledge base regarding the benefits of doing this, alongside the issues regarding truly integrated models - the 'holy grail'. The main conceptual difficulties lie in designing sub-components and their interactions so that they represent the real-world complexity without overwhelming the model with impenetrable detail. This process is both enriched and made more challenging by the combination of disciplines involved. The work required a marriage of an OR stakeholder-driven approach, with the 'empirical eye' of social statisticians and the micro-level theories of complexity science. Social statistics helped make informed decisions on where mechanisms could be abstracted from relationships in empirical data, rather than having the causal mechanisms modeled explicitly. However, the latter is a strong current in complexity science, and promises to help in better modeling individuals' adaptation to changing social and technological contexts, which the scenarios explored here represent. Future work may therefore move further towards this style of theory-driven modeling, à la Noble et al. (2012).

\section{ACKNOWLEDGMENTS}

We gratefully acknowledge the many helpful discussions with the remaining members of the CLC research team not named as authors on this paper (please see www.southampton.ac.uk/clc for a full list of the project team). This work was supported by the EPSRC under grant EP/H021698/1. Finally, we would like to express our gratitude to all the staff at the Southampton Eye Clinic.

\section{REFERENCES}

Access Economics PTY Limited. 2009. Future sight loss UK (1): The economic impact of partial sight and blindness in the UK adult population. RNIB.

Age-Related Eye Disease Study Research, G. 2000. "Risk factors associated with age-related macular degeneration - A case-control study in the age-related eye disease study: age-related eye disease study report number 3." Ophthalmology 107(12): 2224-2232.

Age-Related Eye Disease Study Research, G. 2005. "A Simplified Severity Scale for Age-Related Macular Degeneration." Archives of Ophthalmology 123(11): 1570-1574.

Brailsford, S. C., M. S. Desai and J. Viana. 2010. "Towards the holy grail: combining system dynamics and discrete-event simulation in healthcare". In Proceedings of the 2010 Winter Simulation Conference, Baltimore. Edited by B. Johansson, S. Jain, J. Montoya-Torres, J. Hugan and E. Yucesan

Brailsford, S. C., M. Evandrou, R. Luff, J. Viana, A. Vlachantoni and R. Willis. 2012. "Using system dynamics to model the social care system: linking demography, simulation and care delivery". In Proceedings of the 2012 Winter Simulation Conference. Edited by C. Laroque, J. Himmelspach, R. Pasupathy, O. Rose and A. M. Uhrmacher

Brody, B. L., A. C. Gamst, R. A. Williams, A. R. Smith, P. W. Lau, D. Dolnak, M. H. Rapaport, R. M. Kaplan and S. I. Brown 2001. "Depression, visual acuity, comorbidity, and disability associated with age-related macular degeneration." Ophthalmology 108(10): 1893-1900.

Brown, M. M., G. C. Brown, J. D. Stein, Z. Roth, J. Campanella and G. R. Beauchamp 2005. "Agerelated macular degeneration: economic burden and value-based medicine analysis." Canadian journal of ophthalmology. 40(3): 277-287.

Care Life Cycle. 2012. "The Care Life Cycle [Project]." Accessed 24 March, 2012, from http://www.southampton.ac.uk/clc. 
Chahal, K. and T. Eldabi. 2008. "Applicability of hybrid simulation to different modes of governance in UK healthcare". In Proceedings of the 2008 Winter Simulation Conference. Edited by S. J. Mason, R. R. Hill, L. Monch et al.

Charles, N. 2007. "Estimates of the number of older people with a visual impairment in the UK." British Journal of Visual Impairment 25(3): 199-215.

Cooper JK, Brailsford SC and Davies RM, Choice of modelling technique for evaluating health care interventions Journal of the Operational Research Society (2007) 58: 168 -176

Davies, R., S. Brailsford, P. Roderick, C. Canning and D. Crabbe 2000. "Using Simulation Modelling for Evaluating Screening Services for Diabetic Retinopathy." The Journal of the Operational Research Society 51(4): 476-484.

Davies, R., P. Roderick, C. Canning and S. Brailsford 2002. "The evaluation of screening policies for diabetic retinopathy using simulation." Diabetic Medicine 19(9): 762-770.

ELSA. 2012. "English Longitudinal Study of Ageing." Accessed 1st June, 2012, from http://www.ifs.org.uk/ELSA.

Fletcher, E. C., R. J. Lade, T. Adewoyin and N. V. Chong 2008. "Computerized Model of Cost-Utility Analysis for Treatment of Age-Related Macular Degeneration." Ophthalmology 115(12): 2192-2198.

Hassell, J. B., E. L. Lamoureux and J. E. Keeffe 2006. "Impact of age related macular degeneration on quality of life." British Journal of Ophthalmology 90(5): 593-596.

Jun, J. B., S. H. Jacobson, J. R. Swisher and Correspondence 1999. "Application of discrete-event simulation in health care clinics: A survey." Journal of the Operational Research Society 50(2): 109-123.

Klein, R., B. E. K. Klein, S. C. Tomany, S. M. Meuer and G.-H. Huang 2002. "Ten-year incidence and progression of age-related maculopathy: The Beaver Dam eye study." Ophthalmology 109(10): 17671779 .

Lotery, A. 2008. "Progress in understanding and treating age-related macular degeneration." Eye 22(6): 739-741.

Minassian, D. C. and A. Reidy. 2009. Future Sight Loss UK 2: An epidemiological and economic model for sight loss in the decade 2010-2020.

Minassian, D. C., A. Reidy, A. Lightstone and P. Desai 2011. "Modelling the prevalence of age-related macular degeneration (2010-2020) in the UK: expected impact of anti-vascular endothelial growth factor (VEGF) therapy." British Journal of Ophthalmology. 10.1136/bjo.2010.195370

Noble, J., E. Silverman, J. Bijak, S. Rossiter, M. Evandrou, S. Bullock, A. Vlachantoni and J. Falkingham. 2012. "Linked lives: The utility of an agent-based approach to modelling partnership and household formation in the context of social care". In Proceedings of the 2012 Winter Simulation Conference. Edited by C. Laroque, J. Himmelspach, R. Pasupathy, O. Rose and A. M. Uhrmacher

Owen, C. G., Z. Jarrar, R. Wormald, D. G. Cook, A. E. Fletcher and A. R. Rudnicka 2012. "The estimated prevalence and incidence of late stage age related macular degeneration in the UK." British Journal of Ophthalmology.

Slakter, J. S. and M. Stur 2005. "Quality of Life in Patients With Age-related Macular Degeneration: Impact of the Condition and Benefits of Treatment." Survey of Ophthalmology 50(3): 263-273.

Stevenson, M. R., P. M. Hart, A.-M. Montgomery, D. W. McCulloch and U. Chakravarthy 2004. "Reduced vision in older adults with age related macular degeneration interferes with ability to care for self and impairs role as carer." British Journal of Ophthalmology 88(9): 1125-1130.

Swinerd, C. and K. R. McNaught 2012. "Design classes for hybrid simulations involving agent-based and system dynamics models." Simulation Modelling Practice and Theory 25(0): 118-133.

The Eye Diseases Prevalence Research Group 2004. "Causes and Prevalence of Visual Impairment Among Adults in the United States." Archives of Ophthalmology 122(4): 477-485.

Williams, R. A., B. L. Brody, R. G. Thomas, R. M. Kaplan and S. I. Brown 1998. "The Psychosocial Impact of Macular Degeneration." Archives of Ophthalmology 116(4): 514-520. 


\section{AUTHOR BIOGRAPHIES}

JOE VIANA is a Research Fellow in the Southampton Management School at the University of Southampton. He received a BSc with Honors in Sport and Health Science with Psychology from the University of Southampton, and MSc and PhD in Management Sciences from the University of Southampton. He is primarily interested in modeling health care systems, and in combining different simulation paradigms at different levels. His email address is J.Viana@soton.ac.uk.

STUART ROSSITER is a Research Fellow on the Care Life Cycle project at the University of Southampton. Originally a mathematician, he worked as a software developer and architect for 10 years before focusing on social simulation via an MSc in Multidisciplinary Informatics (Leeds University), and a PhD in the simulation of electricity markets (Strathclyde University, ongoing). He is interested in general issues of modeling and simulation methodology, particularly in processes, software frameworks and agentbased modelling. His email address is S.Rossiter@soton.ac.uk.

ANDREW 'AMOS' CHANNON is a Lecturer in Demography in the Division of Social Statistics and Demography at the University of Southampton. After a BSc at the University of Durham in Psychology he was an IT Project Manager for 6 years before completing an MSc and PhD in Social Statistics at the University of Southampton. His main research interests are in child health in sub-Saharan Africa and population health in the Middle East, but also researches in health systems around the world, especially related to the supply of health care. His email address is a.r.channon@soton.ac.uk.

SALLY C. BRAILSFORD is Professor of Management Science at the University of Southampton, UK. She received a BSc in Mathematics from the University of London, and MSc and PhD in Operational Research from the University of Southampton. Her research interests include simulation modeling methodologies, system dynamics, health service research and disease modeling, and the modeling of human behavior in healthcare systems. She is chair of the European Working Group on OR Applied to Health Services (ORAHS) and is an Editor-in-Chief of Health Systems. She is on the editorial boards of Health Care Management Science, the Journal of Simulation and the Flexible Services \& Manufacturing Journal. Her email address is s.c.brailsford@soton.ac.uk.

ANDREW LOTERY is Professor of Ophthalmology in the Faculty of Medicine at the University of Southampton. He runs an integrated laboratory and clinical ophthalmic research program, dividing his week between directing vision research in the Gift of Sight research laboratories at The University of Southampton and his clinical responsibilities seeing patients in Southampton Eye Unit. His special interests are in medical retina with major interests in age related macular degeneration and ophthalmic genetics; stem cell \& synthetic biology repair, and gene therapy. As well as being Editor in Chief of the journal Eye he and his team have published in high impact journals such as Nature Genetics, the Lancet and the New England Journal of Medicine. His email address is a.j.lotery@soton.ac.uk. 